\title{
ANALYTICAL SOLUTION OF A FRACTIONAL MODEL OF FLUID FLOW THROUGH NARROWING SYSTEM IN TERMS OF MITTAG-LEFFLER FUNCTION
}

\author{
A. CHOUDHARY* \\ Department of Mathematics, Arya Institute of Engineering and Technology \\ Kukus, Jaipur-302028, Rajasthan, INDIA \\ E-mail: choudhary.anupama9@gmail.com \\ D. KUMAR \\ Department of Mathematics, University of Rajasthan \\ Jaipur-302004, Rajasthan, INDIA \\ E-mail: devendra.maths@gmail.com \\ J. SINGH \\ Department of Mathematics, JECRC University \\ Jaipur-303905, Rajasthan, INDIA \\ E-mail: jagdevsinghrathore@gmail.com
}

\begin{abstract}
In this work, we discuss a fractional model of a flow equation in a simple pipeline. Pipeline narrowing is a crucial aspect in drinking water distribution processes, sewage system and in oil-well schemes. The solution of the mathematical model is determined with the aid of the Sumudu transform and finite Hankel transform. The results derived in the current study are in compact and graceful forms in terms of the Mittag-Leffler type function, which are convenient for numerical and theoretical evaluation.
\end{abstract}

Key words: narrowing system, generalized Caputo fractional derivative, Sumudu transform, finite Hankel transform, Bessel function, Mittag-Leffler function.

2010 Mathematics Subject Classification: 35R11, 33E12, 33C10.

\section{Introduction}

Fluid mechanics investigates fluid motion that contains forces of action and reaction, i.e., forces which cause acceleration and forces which resist acceleration. For all flows, we suppose that the pipe is entirely filled with the fluid being transported. So, we do not take a concrete pipe through which rainwater flows without entirely filling the pipe; these kinds of flows are known as open-channel flows. The difference between the pipe flow and an open-channel flow is in the fundamental component that affects the flow. In open-channel flows, gravity is only the driving force, i.e., the water flows downwards. In pipe flows, gravity may be crucial as the pipe is not necessarily be horizontal, but the key driving force is generally to be a pressure gradient through the pipe. If the flow is not fully developed in the pipe, than the pressure difference is not possible to be maintained.

For an incompressible flow, the continuity and Navier-Stokes equations (NSEs) are [1]

\footnotetext{
* To whom correspondence should be addressed
} 


$$
\begin{aligned}
& \nabla \cdot \bar{V}=\frac{1}{r} \frac{\partial}{\partial r}(r U)+\frac{1}{r} \frac{\partial V}{\partial \theta}+\frac{\partial W}{\partial z}=0, \\
& \rho\left(\frac{D \bar{V}}{D t}\right)=-\nabla p+\mu \nabla^{2} \bar{V} .
\end{aligned}
$$

Equation (1.2) can be reduced to cylindrical coordinates $r, \theta$ and $z$ directions below as

$$
\begin{aligned}
& \frac{\partial U}{\partial t}+U \frac{\partial U}{\partial r}+\frac{V}{r} \frac{\partial U}{\partial \theta}+W \frac{\partial U}{\partial z}-\frac{V^{2}}{r}= \\
& =-\frac{1}{\rho} \frac{\partial p}{\partial r}+\frac{\mu}{\rho}\left(\frac{\partial}{\partial r}\left(\frac{1}{r} \frac{\partial(r U)}{\partial r}\right)+\frac{1}{r^{2}} \frac{\partial^{2} U}{\partial \theta^{2}}+\frac{\partial^{2} U}{\partial z^{2}}-\frac{2}{r^{2}} \frac{\partial V}{\partial \theta}\right) \\
& \frac{\partial V}{\partial t}+U \frac{\partial V}{\partial r}+\frac{V}{r} \frac{\partial V}{\partial \theta}+W \frac{\partial V}{\partial z}+\frac{U V}{r}= \\
& =-\frac{1}{\rho} \frac{1}{r} \frac{\partial p}{\partial \theta}+\frac{\mu}{\rho}\left(\frac{\partial}{\partial r}\left(\frac{1}{r} \frac{\partial(r V)}{\partial r}\right)+\frac{1}{r^{2}} \frac{\partial^{2} V}{\partial \theta^{2}}+\frac{\partial^{2} V}{\partial z^{2}}+\frac{2}{r^{2}} \frac{\partial U}{\partial \theta}\right), \\
& \frac{\partial W}{\partial t}+U \frac{\partial W}{\partial r}+\frac{V}{r} \frac{\partial W}{\partial \theta}+W \frac{\partial W}{\partial z}=-\frac{1}{\rho} \frac{\partial p}{\partial z}+\frac{\mu}{\rho}\left(\frac{1}{r} \frac{\partial}{\partial r}\left(r \frac{\partial W}{\partial r}\right)+\frac{1}{r^{2}} \frac{\partial^{2} W}{\partial \theta^{2}}+\frac{\partial^{2} W}{\partial z^{2}}\right)
\end{aligned}
$$

The NSEs are helpful because they interpret the process of several aspects of academic and monetary significance. They are utilized to model the weather, fluid flow in a pipe and air flow about a wing. The NSEs in their wide and simplest forms assists with the study of fluid flow. A number of researchers have also investigated fluid flow by using different mathematical resources [2-10]. In this work, we developed a fractional model for the study of fluid flow in narrowing system by using Eq.(1.2) with the help of equation of continuity and applying the Sumudu and Hankel transforms, which generate the solution for the time fractional NSE in a circular cylinder. The notable and crucial advantage of utilization of fractional derivatives in mathematical modeling is their non-local nature. It is established fact that the integer order differential operator is a local operator but the differential operator of fractional order is non-local. This implies that the next state of a dynamical process depends not only upon its current state but also upon all of its past states. Consequently the fractional modeling is more reliable and effective it is one reason why a fractional order models have gained more and more popularity in science and technology [11-27].

The key aim of this work is development of a solution of a fractional model of a flow equation in a simple pipeline. The Sumudu transform and finite Hankel transform are utilized to achieve the solution of the fractional problem. The results are derived in compact and graceful forms in terms of the extended MittagLeffler (ML) function, which are highly systematic and effective for numerical simulation.

\section{Preliminary results}

The Sumudu transform (ST) of a function $f(t)$, determined for all real numbers $t \geq 0$, is the function $F_{S}(u)$, defined by [28-31] 


$$
S\{f(t)\}=\bar{F}(u)=G(u)=\int_{0}^{\infty}(1 / u) e^{-t / u} f(t) d t
$$

We utilize the following results $[32,33]$ as

$$
S^{-1}\left[u^{\mu-1}\left(1-\omega u^{\rho}\right)^{-\lambda}\right]=t^{\mu-1} E_{\rho, \mu}^{\lambda}\left(\omega t^{\rho}\right), \quad \lambda, \rho, \mu \in \mathbb{C}, \mathfrak{R}(\rho)>0
$$

where $(\lambda)_{k}$ is the parameter symbol and is defined as

$$
(\lambda)_{0}=1, \quad(\lambda)_{k}=\lambda(\lambda+1) \ldots(\lambda+k-1) ; \quad k=1,2, \ldots
$$

The zero order Bessel function $J_{0}(x)$ [34] is defined as

$$
J_{o}(x)=\sum_{n=0}^{\infty} \frac{(-1)^{n}}{(n !)^{2}}\left(\frac{x}{2}\right)^{2 n}
$$

The zero order finite Hankel transform [35] is defined as

$$
H_{o}\{f(r)\}=\tilde{f}_{0}\left(\xi_{n}\right)=\int_{0}^{R} r f(r) J_{o}\left(r \xi_{n}\right) d r
$$

where $\xi_{n}$ are the roots of the equation $J_{o}\left(R \xi_{n}\right)=0$.

We also use the below result

$$
f(r)=\int_{0}^{R} \xi_{n} \tilde{f}_{0}\left(\xi_{n}\right) J_{o}\left(r \xi_{n}\right) d \xi_{n}
$$

An extension of the ML function [36, 37]

$$
E_{\alpha}(z)=\sum_{n=0}^{\infty} \frac{z^{n}}{\Gamma(n \alpha+l)}, \quad(\alpha \epsilon C, \mathbb{R}(\alpha)>0),
$$

was given in [38] in the general form

$$
E_{\alpha, \beta}(z)=\sum_{n=0}^{\infty} \frac{z^{n}}{\Gamma(n \alpha+\beta)}, \quad(\alpha, \beta \epsilon C, \mathbb{R}(\alpha)>0)
$$

also derived in [39] in the subsequent integral

$$
\int_{0}^{\infty} e^{-s t} t^{\beta-1} \frac{d^{k}}{d z^{k}} E_{\alpha, \beta}\left(x t^{\alpha}\right) d t=\frac{k ! s^{\alpha-\beta}}{\left(s^{\alpha}-x\right)^{k+1}} .
$$


The 3 parameter ML function was defined by Prabhakar [40] and a detailed study was conducted by Garra and Garrappa [41]. The function is expressed as follows

$$
E_{\alpha, \beta}^{\gamma}(z)=\sum_{n=0}^{\infty} \frac{(\gamma)_{n} z^{n}}{\mathrm{n} ! \Gamma(n \alpha+\beta)}, \quad(\alpha, \beta, \gamma \epsilon C, \mathbb{R}(\alpha)>0)
$$

The fractional derivative of order $\alpha>0$ is suggsted by Caputo [11] in the form

$$
\begin{aligned}
& { }_{0}^{c} D_{x}^{\alpha} f(x)=\frac{1}{\Gamma(m-\alpha)} \int_{0}^{x} \frac{f^{(m)}(\tau)}{(x-\tau)^{\alpha-m+1}} d \tau, \quad m-1<\alpha<m \\
& { }_{0}^{c} D_{x}^{\alpha} f(x)=\frac{d^{m} f(t)}{d t^{m}}, \quad \text { if } \quad \alpha=m ; m \epsilon N
\end{aligned}
$$

where $\frac{d^{m} f(t)}{d t^{m}}$ is the $m^{t h}$ derivative of order $m$ of the function $f(t)$ with respect to $t$. The Sumudu transform of the above derivative of arbitrary order is presented in [42]

$$
S\left[{ }_{0}^{c} D_{x}^{\alpha} f(x) ; s\right]=u^{-\alpha} \bar{f}(s)-\sum_{k=0}^{m-1} u^{-\alpha+k} f^{(k)}(0+), \quad m-1<\alpha \leq m .
$$

An extension of the Caputo fractional operator Eq.(2.10) is suggested in [43], by using a right-sided fractional operator of 2 parameters of order $0<\alpha<1$ and $0 \leq \beta \leq 1$

$$
{ }_{0} D_{a+}^{\alpha, \beta} f(x)=I_{a+}^{\beta(1-\alpha)} \frac{d}{d x}\left(I_{a+}^{(I-\beta)(I-\alpha)}\right) f(x) .
$$

If we set $\beta=1$, Eq.(2.12) yields the Caputo fractional operator (2.10).

The ST of the extended fractional derivative $[43,44]$ is presented as

$$
S\left[{ }_{0} D_{x}^{\alpha, \beta} f(x) ; s\right]=u^{-\alpha} \bar{f}(s)-u^{-\beta(\alpha-1)+1} I_{0+}^{(1-\beta)(1-\alpha)} f(0+) ; \quad 0<\alpha \leq 1
$$

where the initial value term

$$
I_{0+}^{(1-\beta)(1-\alpha)} f(0+)
$$

contains the Riemann-Liouville (RL) fractional integral operator of order $(1-\beta)(1-\alpha)$ evaluated in the limit as $x \rightarrow 0+$. In any one want to see detailed results of this operator refer the work in [44]. 


\section{Formulation of problem}

We consider a long circular cylinder in which the fluid is at rest initial position and a fixed pressure gradient fluid is set into motion (constant $\rho$ and $\mu$ ) is inflicted along the axis of cylinder. Assume $\mathbb{Z}$ be the direction of the axis of the cylinder along which the fluid flows and suppose the $r$ be the radial direction departing from the $\mathbb{Z}$-axis, due to the fluid flow is produced and symmetric about the axis. Here, we consider that on the wall of the cylinder there are some deposition of thickness $\Delta$, which results in the narrowing system and holds the following equation of deposition of thickness

$$
R=R_{0}-\frac{\Delta}{2}\left(1+\cos \cos \frac{\pi z}{z_{0}}\right)
$$

where $\Delta$ is the thickness of deposition and $R_{0}$ is standing for the distance from the axis of cylindrical boundary and $z$ is the distance from $z=0$ to the point of calculation $P$.

If

$$
z=0 \text { then } R=R_{0}-\Delta \text { and if } z=z_{0} \text { then } R=R_{0} .
$$

Since velocities $U$ and $V$ are zero and pressure depends on $z$ then we get the result presented in Eqs (1.1)(1.3)

$$
\frac{\partial W}{\partial t}+W \frac{\partial W}{\partial r}=-\frac{1}{\rho} \frac{\partial p}{\partial z}+\frac{\mu}{\rho}\left(\frac{1}{r} \frac{\partial}{\partial r}\left(r \frac{\partial W}{\partial r}\right)+\frac{1}{r^{2}} \frac{\partial^{2} W}{\partial \theta^{2}}+\frac{\partial^{2} W}{\partial z^{2}}\right)
$$

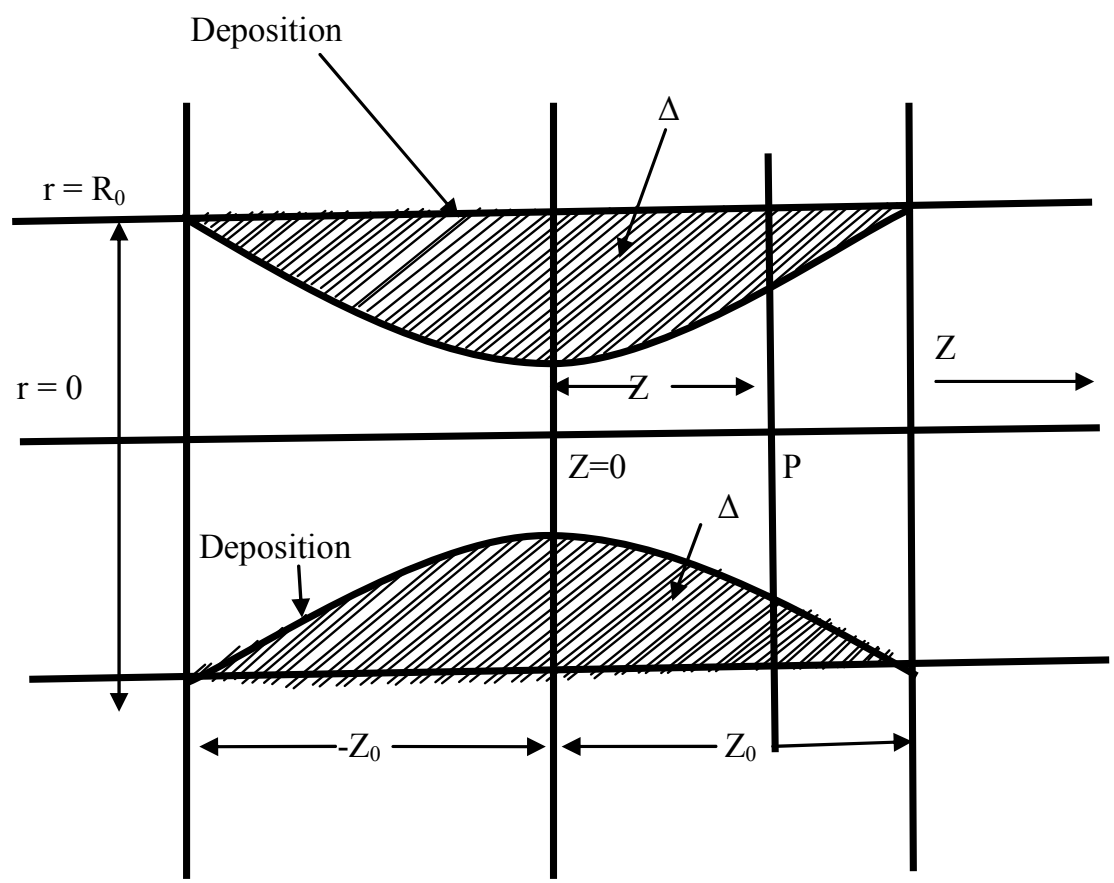

Fig.1. Schematic diagram of the flow problem. 
We assume that the velocity component is invariant in the $\theta$ and $z$ directions, then the above equation becomes

$$
\frac{\partial W}{\partial t}+W \frac{\partial W}{\partial z}=-\frac{1}{\rho} \frac{\partial p}{\partial z}+\frac{\mu}{\rho}\left(\frac{1}{r} \frac{\partial}{\partial r}\left(r \frac{\partial W}{\partial r}\right)\right)
$$

Now, using the equation of continuity

$$
\frac{\partial W}{\partial z}=0
$$

the above equation becomes

$$
\rho \frac{\partial W}{\partial t}=P+\mu\left(\frac{\partial^{2} W}{\partial r^{2}}+\frac{1}{r} \frac{\partial W}{\partial r}\right)
$$

where $\mu$ is the dynamic viscosity and

$$
P=-\frac{\partial p}{\partial z}
$$

In present paper, we take the fractional model of Eq. (3.2) in the following form

$$
\rho D_{t}^{\alpha, \beta} W(r, t)=P+\mu\left(\frac{\partial^{2} W}{\partial r^{2}}+\frac{1}{r} \frac{\partial W}{\partial r}\right),
$$

subject to initial and constraint conditions are taken as

$$
\left\{\begin{array}{c}
\left(I_{0}^{(1-\alpha)(1-\beta)} W\right)(r, 0)=0 \\
W(R, t)=0 \\
W(0, t) \text { is finite. }
\end{array}\right.
$$

\section{Solution of problem}

To evaluate the solution of the problem, we use the integral transform as follows

$$
\tilde{W}\left(\xi_{n}, t\right)=H_{0}(W(r, t))=\int_{0}^{R} r W(r, t) J_{0}\left(r \xi_{n}\right) d r
$$


where $\xi_{n}$ are the roots of $J_{0}\left(R \xi_{n}\right)=0$. By using the recurrence relation of the Bessel function holds

$$
\frac{d}{d x}\left\{x^{n} J_{n}(x)\right\}=x^{n} J_{n-1}(x) ; \quad n \geq 0
$$

Putting $n=1$ and $x=r \xi_{n}$, we get

$$
\left[r \xi_{n} J_{l}\left(r \xi_{n}\right)\right]_{0}^{R}=\int_{0}^{R} r \xi_{n}^{2} J_{0}\left(r \xi_{n}\right) d r
$$

or $\quad \int_{0}^{R} r J_{0}\left(r \xi_{n}\right) d r=\frac{R}{\xi_{n}} J_{l}\left(R \xi_{n}\right)$.

We take the zero order finite Hankel transform Eq.(2.4) of Eq.(3.3) and using Eqs (3.4), (4.1) and (4.2), we get

$$
\rho D_{t}^{\alpha, \beta} \tilde{W}=\frac{P R J_{l}\left(R \xi_{n}\right)}{\xi_{n}}-\mu \xi_{n}^{2} \tilde{W}
$$

By using the ST on both sides of Eq.(4.3) and using Eqs (2.1), (2.13) and (3.4), we get the results

$$
\rho u^{-\alpha} \overline{\tilde{W}}\left(\xi_{n}, u\right)-u^{-\beta(\alpha-I)+1} I_{0+}^{(l-\beta)(I-\alpha)} \tilde{W}\left(\xi_{n}, 0\right)=\frac{P R J_{I}\left(R \xi_{n}\right)}{\xi_{n}}-\mu \xi_{n}{ }^{2} \overline{\tilde{W}}
$$

It yields

$$
\overline{\tilde{W}}\left(\xi_{n}, u\right)=\frac{P R J_{1}\left(R \xi_{n}\right)}{\rho \xi_{n}\left[u^{-\alpha}+\frac{\mu}{\rho} \xi_{n}{ }^{2}\right]} .
$$

Now, making use of the inverse ST on both sides of Eq.(4.5), and with the aid of Eq.(2.2), which is given

$$
\tilde{W}\left(\xi_{n}, t\right)=\frac{P R J_{1}\left(R \xi_{n}\right)}{\rho \xi_{n}} t^{\alpha} E_{\alpha, \alpha+1}\left(-\frac{\mu}{\rho} \xi_{n}{ }^{2} t^{\alpha}\right) .
$$

On utilizing the inverse finite Hankel transform on both sides Eq.(4.6), and employing Eq.(2.5), we achieve the subsequent result 


$$
W(r, t)=\frac{2}{R^{2}} \sum_{n=1}^{\infty}\left\{\frac{P R J_{1}\left(R \xi_{n}\right)}{\rho \xi_{n}} t^{\alpha} E_{\alpha, \alpha+1}\left(-\frac{\mu}{\rho} \xi_{n}{ }^{2} t^{\alpha}\right)\right\} \frac{J_{0}\left(r \xi_{n}\right)}{J_{l}{ }^{2}\left(R \xi_{n}\right)} .
$$

If we set $\alpha=1$ and $\beta=1$ then we achieve the below result recently obtained in [45]

$$
W(r, t)=\frac{2}{R^{2}} \sum_{n=1}^{\infty}\left\{\frac{P R J_{1}\left(R \xi_{n}\right)}{\rho \xi_{n}} t E_{1,2}\left(-\xi_{n}{ }^{2} \frac{\mu}{\rho} t\right)\right\} \frac{J_{0}\left(r \xi_{n}\right)}{J_{l}^{2}\left(R \xi_{n}\right)}
$$

We can easily verify that

$$
t E_{1,2}(m t)=\frac{1}{m}\left[e^{m t}-1\right]
$$

And using the above result in Eq.(4.8)

$$
W(r, t)=-\frac{2}{R^{2}} \sum_{n=1}^{\infty}\left\{\frac{P R J_{1}\left(R \xi_{n}\right)}{\rho \xi_{n}} \frac{1}{\xi_{n}{ }^{2} \frac{\mu}{\rho}}\left(e^{-\xi_{n}{ }^{2} \frac{\mu}{\rho} t}-1\right)\right\} \frac{J_{0}\left(r \xi_{n}\right)}{J_{l}^{2}\left(R \xi_{n}\right)} .
$$

We can write the above result as

$$
W(r, t)=\frac{2 P}{\mu R} \sum_{n=1}^{\infty}\left\{\left(1-e^{-\xi_{n}{ }^{2} \frac{\mu}{\rho} t}\right)\right\} \frac{J_{0}\left(r \xi_{n}\right)}{\xi_{n}{ }^{3} J_{l}\left(R \xi_{n}\right)},
$$

or

$$
W(r, t)=\frac{P}{4 \mu}\left(R^{2}-r^{2}\right)-\frac{2 P}{\mu R} \sum_{n=1}^{\infty} \frac{J_{0}\left(r \xi_{n}\right)}{\xi_{n}^{3} J_{l}\left(R \xi_{n}\right)} e^{\left(-\xi_{n}{ }^{2} \frac{\mu}{\rho} t\right)} .
$$

If we are taking the limit $t \rightarrow \infty$, both sides of Eq.(4.10), then we have

$$
W(r, t)=\frac{P}{4 \mu}\left(R^{2}-r^{2}\right)
$$

\section{Conclusions}

In this work, we have studied a mathematical model of a flow equation in a simple pipeline with fractional order. The study of pipeline narrowing is a crucial and fundament aspect in drinking water distribution processes, sewage system and in oil-well schemes. The analytical solution for the fractional modeling of fluid flow through a narrowing system is obtained with the aid of the Sumudu and finite Hankel transforms and their inverse after evaluating the concerned formulae for fractional calculus. The solution has 
been developed in a closed form in terms of the ML function and Bessel's function. Therefore the determined solution is very suitable for numerical simulation purpose. The results computed by us are general in nature and contain the known results existing in the literature. Hence, we can conclude that the generalized Caputo fractional operator is very useful in mathematical modeling of natural phenomena and the suggested technique can be used to examine such type of fractional models.

\section{Nomenclature}

$$
\begin{array}{cl}
D & - \text { substantial derivative } \\
\bar{V} & - \text { velocity vector } \\
t & - \text { time } \\
\rho & - \text { density } \\
p & - \text { pressure } \\
v & - \text { kinematic viscosity } \\
\mu & - \text { dynamic viscosity }
\end{array}
$$

\section{References}

[1] Landau L.D. and Lifshitz E.M. (1959): Fluid Mechanics. - Pergamon Press.

[2] Acheson D.J. (1990): Elementary Fluid Dynamics. - Oxford Applied Mathematics and Computing Science Series, Oxford University Press, ISBN 0-19-859679-0.

[3] Batchelor G.K. (1967): An Introduction to Fluid Dynamics. - Cambridge University Press, ISBN 0-521-66396-2.

[4] Upadhyay V. (2000): Some Phenomena in Two Phase Blood Flow. - Ph. D. Thesis, Central University, Allahabad.

[5] Debnath L. (1976): On a micro - continuum model of pulsatile blood flow. - Acta Mechanica, vol.24, pp.165-177.

[6] Jones D.S. and Sleeman B.D. (1976): Differential Equation and Mathematical Biology. - 6 G.A. and V.

[7] Upadhyay V. and Pandey P.N. (1999): Newtonian Model of two phase blood flow in aorta and arteries proximate to the heart. - Proc. of Third Con. of Int. Acad. Phy. Sci.

[8] Upadhyay V. and Pande P.N. (1999): A power law model of two phase blood flow in arteries remote form the heart. - Proc. of Third Con. of Int. Acad. Phy. Sci..

[9] Singh P. and Upadhyay K.S. (1985): A new approach for the shock propagation in two - phase system. - Nat. Acad. Sci. Letters No.2.

[10] Debnath L. (1973): On transient flows in non-Newtonian liquids. - Tensor N.S., vol.27, No.2, pp.257-264.

[11] Caputo M. (1967): Linear Models of Dissipation whose $Q$ is almost Frequency Independent-II. - Geophysical J. of the Royal Astronomical Soc., vol.13, pp.529-539. (Reprinted in : Fract. Calc. Appl. Anal., 11 no. 1 (2008), 314.

[12] Hilfer R. (ed.) (2000): Applications of Fractional Calculus in Physics. - World Scientific Publishing Company, Singapore-New Jersey-Hong Kong, pp.87-130.

[13] Podlubny I. (1999): Fractional Deferential Equations (An Introduction to Fractional Derivatives, Fractional Deferential Equations, to Methods of Their Solution and some of Their Applications). - Academic Press, San Diego.

[14] Miller K.S. and Ross B. (1993): An Introduction to the Fractional Calculus and Fractional Differential Equations. - New York: Wiley.

[15] Samko S.G., Kilbas A.A. and Marichev O.I. (1993): Fractional Integrals and Derivatives: Theory and Applications. - Switzerland: Gordon and Breach Science Publishing. 
[16] Ansari A. (2015): On finite fractional Sturm-Liouville transforms. - Integral Transforms and Special Functions, vol.26, No.1, pp.51-64.

[17] Kumar D., Singh J. and Baleanu D. (2017): A hybrid computational approach for Klein- Gordon equations on Cantor sets. - Nonlinear Dynamics, vol.87, pp.511-517.

[18] Srivastava H.M., Kumar D. and Singh J. (2017): An efficient analytical technique for fractional model of vibration equation. - Applied Mathematical Modelling, vol.45, pp.192-204.

[19] Kumar D., Agarwal R.P. and Singh J. (2017): A modified numerical scheme and convergence analysis for fractional model of Lienard's equation. - Journal of Computational and Applied Mathematics, http://doi.org/10.1016/j.cam.2017.03.0.11

[20] Kumar D., Singh J. and Baleanu D. (2017): A new analysis for fractional model of regularized long-wave equation arising in ion acoustic plasma waves. - Mathematical Methods in the Applied Sciences, DOI:10.1002/mma.4414.

[21] Singh J., Rashidi M.M., Swroop R. and Kumar D. (2016): A fractional model of a dynamical Brusselator reactiondiffusion system arising in triple collision and enzymatic reactions. - Nonlinear Engineering - Modeling and Application, vol.5, No.4, pp.277-285.

[22] Podlubny I. (1999): Fractional differential equations. - Academic Press, San Diego, Calif, USA, vol.198, pp.340 pages.

[23] Kilbas A.A., Srivastava H.M and Trujillo J.J. (2006): Theory and applications of fractional differential equations. - Elsevier, Amsterdam, vol.20), 540 pages.

[24] Srivastava H.M., Golmankhaneh A.K., Baleanu D. and Yang X.J. (2014): Local Fractional Sumudu Transform with Application to IVPs on Cantor Sets. - Abst. Appl. Anal., article ID 620529, 7 pages.

[25] Shukla A.K. and Prajapati J.C. (2007): On some properties of class of polynomials suggested by mittal. Universidad Cat'olica del Norte Progyecciones (Antofagasta-Chile), vol.26, No.2, pp.145-156.

[26] Shukla A.K. and Prajapati J.C. (2008): On generalized Mittag-Leffler type function and generated integral operator. - Mathematical Sciences Research Journal, vol.12, No.12, pp.283-290.

[27] Prajapati J.C. and Nathwani B.V. (2015): Fractional calculus of a unified Mittag-Leffler function. - Ukrainian Mathematical Journal, vol.66, No.8, pp.1267-1280.

[28] Watugala G.K. (1993): Sumudu transform, a new integral transform to solve differential equations and control engineering problems. - International Journal of Mathematical Education in Science and Technology, vol.24, pp.35-43.

[29] Weerakoon S. (1994): Application of Sumudu transform to partial differential equations. - International Journal of Mathematical Education in Science and Technology, vol.25, pp.277-283.

[30] Asiru M.A. (2001): Sumudu transform and the solution of integral equation of convolution type. - International Journal of Mathematical Education in Science and Technology, vol.32, pp.906-910.

[31] Belgacem F.B.M. and Karaballi A.A. (2005): Sumudu transform fundamental properties investigations and applications. - International J. Appl. Math. Stoch. Anal., pp.1-23.

[32] Chaurasia V.B.L. and Singh J. (2011): Application of Sumudu transform in fractional kinetic equations. - Gen. Math. Notes, vol.2, No.1, pp.86-95.

[33] Belgacem F.B.M. and Al-Shemas E.H.N. (2014): Towards a Sumudu based estimation of large scale disasters environmental fitness changes adversely affecting population dispersal and persistence. - AIP Conf. Proc. 1637, (1442); doi: 10.1063/1.4907311.

[34] Rainville E.D. (1960): Special Functions. - New York: The Macmillan Company.

[35] Debnath L. (1995): Integral Transforms and their Applications. - New York-London-Tokyo: CRC Press.

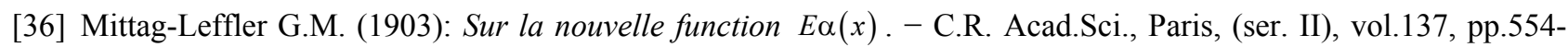
558. 
[37] Mittag-Leffler G.M. (1905): Sur la representatin analytique d'une branche uniforme d'une function monogene. Acta Math., vol.29, pp.101-181.

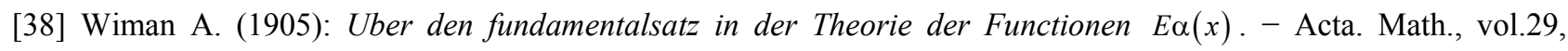
pp.191-201.

[39] Shukla K. and Prajapat J.C. (2007): On a generalization of Mittag-Leffler function and its properties. - J. Math. Anal. Appl., vol.336, pp.797-811.

[40] Prabhakar T.R. (1971): A singular integral equation with a generalized Mittag-Leffler function in the kernel. Yokohama Math. J., vol.19, pp.7-15.

[41] Garra R. and Garrappa R. (2018): The Prabhakar or three parameter Mittag-Leffler function: Theory and application. - Communications in Nonlinear Science and Numerical Simulation, vol.56, pp.314-329.

[42] Chaurasia V.B.L. and Singh J. (2010): Application of Sumudu transform in Schodinger equation occurring in quantum mechanics. - Applied Mathematical Sciences, vol.4, No.57, pp.2843-2850.

[43] Belgacem F.B.M., Karaballi A.A. and Kalla S.L. (2003): Analytical investigations of the Sumudu transform and applications to integral production equations. - Mathematical Problems in Engineering, vol.3, pp.103-118.

[44] Tomovski Z., Hilfer R. and Srivastava H.M. (2010): Fractional and operational calculus with generalized fractional derivative operators and Mittag-Leffler type functions. - Integral Transforms Spec. Funct., vol.21, No.11, pp.797-814.

[45] Patel A.D., Salehbhai I.A., Banerjee J., Katiyar V.K. and Shukla A.K. (2012): An analytical solution of fluid flow through narrowing systems. - Italian Journal of Pure and Applied Mathematics, vol.29, pp.63-70.

Received: July 10, 2018

Revised: December 7, 2019 